\title{
The evolving role of radiotherapy in the management of small cell lung cancer
}

\author{
Mark V. Mishra ${ }^{1}$, Alexander V. Louie ${ }^{2}$, Vinai Gondi $^{3}$, Ben Slotman ${ }^{4}$ \\ ${ }^{1}$ University of Maryland School of Medicine, Baltimore, Maryland, USA; ${ }^{2}$ London Health Sciences Centre, ON, Canada; ${ }^{3}$ Northwestern University \\ Feinberg School of Medicine, Chicago, Illinois, USA; ${ }^{4}$ UU University Medical Center, Amsterdam, The Netherlands \\ Contributions: (I) Conception and design: All authors; (II) Administrative support: None; (III) Provision of study materials or patients: None; (IV) \\ Collection and assembly of data: None; (V) Data analysis and interpretation: None; (VI) Manuscript writing: All authors; (VII) Final approval of \\ manuscript: All authors. \\ Correspondence to: Mark V. Mishra, MD. Assistant Professor, Department of Radiation Oncology, University of Maryland School of Medicine, \\ Baltimore, Maryland, USA. Email: mmishra@umm.edu.
}

\begin{abstract}
Small cell lung cancer (SCLC) represents a small but significant subset of newly diagnosed lung cancers. In spite of being both chemo- and radiation-sensitive, SCLC has a high-propensity for recurrence after treatment. Although systemic therapy plays a central role in the management of patients with SCLC, many of the advances in overall survival for patients with SCLC have directly related to the use of radiation therapy. The objective of this review is to discuss the key radiation therapy clinical trials that have defined the current standard-of-care treatment for SCLC, and to review ongoing advances in radiation therapy that may further advance outcomes for patients with SCLC.
\end{abstract}

Keywords: Small cell lung cancer (SCLC); limited stage; extensive stage; radiation; b.i.d. radiation; stereotactic body radiation therapy (SBRT); prophylactic cranial irradiation (PCI); hippocampal avoidance (HA)

Submitted Jan 08, 2018. Accepted for publication Jun 14, 2018.

doi: $10.21037 /$ jtd.2018.06.98

View this article at: http://dx.doi.org/10.21037/jtd.2018.06.98

\section{Introduction}

Small cell lung cancer (SCLC) is an uncommon but aggressive subset of primary lung cancers that accounts for nearly $15 \%$ of newly diagnosed lung cancers in the United States (US). The American Cancer Society estimates that there will be approximately 35,000 new cases of SCLC in the US in 2018 (1).

As recent as the 1940s-1950s, SCLC had not yet recognized as a distinct entity compared to other types of lung cancer. Treatment for patients consisted of surgery for operable patients, radiation therapy (RT) for inoperable cases, and nitrogen mustard for situations where systemic therapy was warranted. SCLC was only first described as a distinct entity from other types of lung cancer in the 1960s in a case report published by Watson and Berg (2), where the authors described the primary objective of their paper was to "protest against the lumpers who would classify these tumors as epidermoid carcinomas". The authors subsequently outlined the distinct clinical progression of "oat cell" cancer and hypothesized that "oat cell cancer of the lung with its low resectability and low curability rate might be better treated by a combination of intensive chemotherapy and supravoltage RT", which is ultimately our standard of care today.

Since this time, significant progress has been achieved in terms of better understanding the etiology and pathogenesis of SCLC (Figure 1). In the modern era, SCLC is described as a small round blue cell tumor (3), with approximately $75 \%$ cases having one or more neuroendocrine markers such as chromograndin, synaptophysin, and virtually all cases reactive for keratin as well as epithelial membrane antigen.

Smoking is well established as the primary risk factor for developing SCLC, with other risk factors including exposure to radon, asbestos, arsenic, as well as polycyclic aromatic hydrocarbons (4). Since the landmark US Surgeon 
General's report on smoking and its' ill effects in 1964 (5), several public health efforts have been successfully initiated to reduce smoking within the American population, which has fortunately resulted in an overall decline in the incidence of SCLC in the US (6). However, the incidence of SCLC has remained fairly constant in the elderly population, likely representing the long-term impact of cigarette smoking in this population that predates Surgeon General's report. Although SCLC remains more common in males than females, overall incidence rates are decreasing faster in males than in females, which mirrors smoking trends in males vs. females. However, females in general have a better prognosis with longer overall survival (OS) than males (7).

Staging of SCLC has also changed significantly over time. The initial landmark staging systems established through the VA Lung Study Group divided SCLC into two groups: limited-stage (about a third of all SCLC patients) vs. extensive stage (about two-thirds of all patients) (8). Limited stage disease was defined as disease that is confined to the ipsilateral hemithorax and within a single radiation portal. All other cases were classified as extensive-stage disease. Further refinement of the staging of SCLC came with the American Joint Committee of Cancer (AJCC) $7^{\text {th }}$ edition, where TNM staging of SCLC was introduced, is identical to that of non-SCLC (NSCLC), and will be further revised with the introduction of the AJCC $8^{\text {th }}$ edition in 2018.

\section{Limited-stage SCLC (LS-SCLC): a historical perspective}

The role of RT for SCLC has been evaluated most extensively for patients with limited-stage disease. Shortly after the first description of SCLC as a distinct pathologic entity from of NSCLC by Watson and Berg in 1962 (2), the first randomized study was initiated by the UK Medical Research Council (MRC) Study to better define the optimal local therapy for patients with $\operatorname{SCLC}(9,10)$. Patients in this study were randomized to either curative-intent RT, using the dose and "technique customarily used by radiotherapist," or surgery. Long-term results demonstrated a significant improvement in OS for patients treated with RT as compared with surgery, with a mean survival of 284 vs. 199 days, respectively. After 5 years of follow-up, there was only one surviving patient in the surgery arm who, "although allocated to a policy of surgery, became too breatbless to be operated upon and was therefore treated with radiotherapy". Since this time, RT has been considered to be the preferred local treatment for SCLC.

Following the MRC study, numerous studies were conducted to evaluate the role of chemotherapeutic agents for treatment of LS-SCLC. The first systemic therapy studies that were conducted showed a benefit to use of single-agent chemotherapy. This was followed by studies in the 1970s that demonstrated a significant benefit with the use of combination chemotherapy compared to single-agent chemotherapy, with an anthracycline-based regimen at that time. Clinical trials in 1980s later established platinumbased chemotherapy as the preferred first-line systemic treatment regimen for SCLC.

Between the 1980s and 1990s, there were multiple studies initiated to help understand the role of RT for patients with limited-stage disease in light of studies showing benefit to chemotherapy (11). During this time period, there were nearly two-dozen North American SCLC randomized studies of which only five studies showed positive findings, and all five were asking an experimental question related to RT.

The first set of studies was done to ask the question: "Does the combination of thoracic radiation and chemotherapy improve outcomes for patients with LS-SCLC?" This was addressed through multiple clinical trials $(12,13)$, with the defining study being the CALGB (12). In this study, patients were randomized to either chemotherapy-alone, chemotherapy followed by RT, or concurrent chemoradiation. The results showed a significant improvement in both progressionfree survival (PFS) and OS, favoring the arms of the study that included RT. A subsequent study conducted in Japan showed that concurrent RT $v s$. sequential chemoradiotherapy was associated with improved outcomes. Ultimately, meta-analyses of studies evaluating chemotherapy alone $v s$. thoracic RT in combination with chemotherapy demonstrated an absolute improvement in 3 -year OS of $5.4 \%(14,15)$.

Following the studies showing a survival benefit of use of RT with chemotherapy, the next set of studies were initiated to answer the question: "What is the optimal timing of thoracic radiation for patients with LS-SCLC?" The largest study was designed to answer this question was conducted by the NCIC group (16). Patients in this study were randomized to receive 6 cycles of chemotherapy with thoracic radiation (40 Gy delivered over 15 fractions) initiated at cycle $2 v s$. cycle 6 of chemotherapy. All patients with stable disease following combination therapy received prophylactic cranial irradiation (PCI). Long-term results demonstrated a benefit in terms of PFS as well as OS for patients treated with RT 


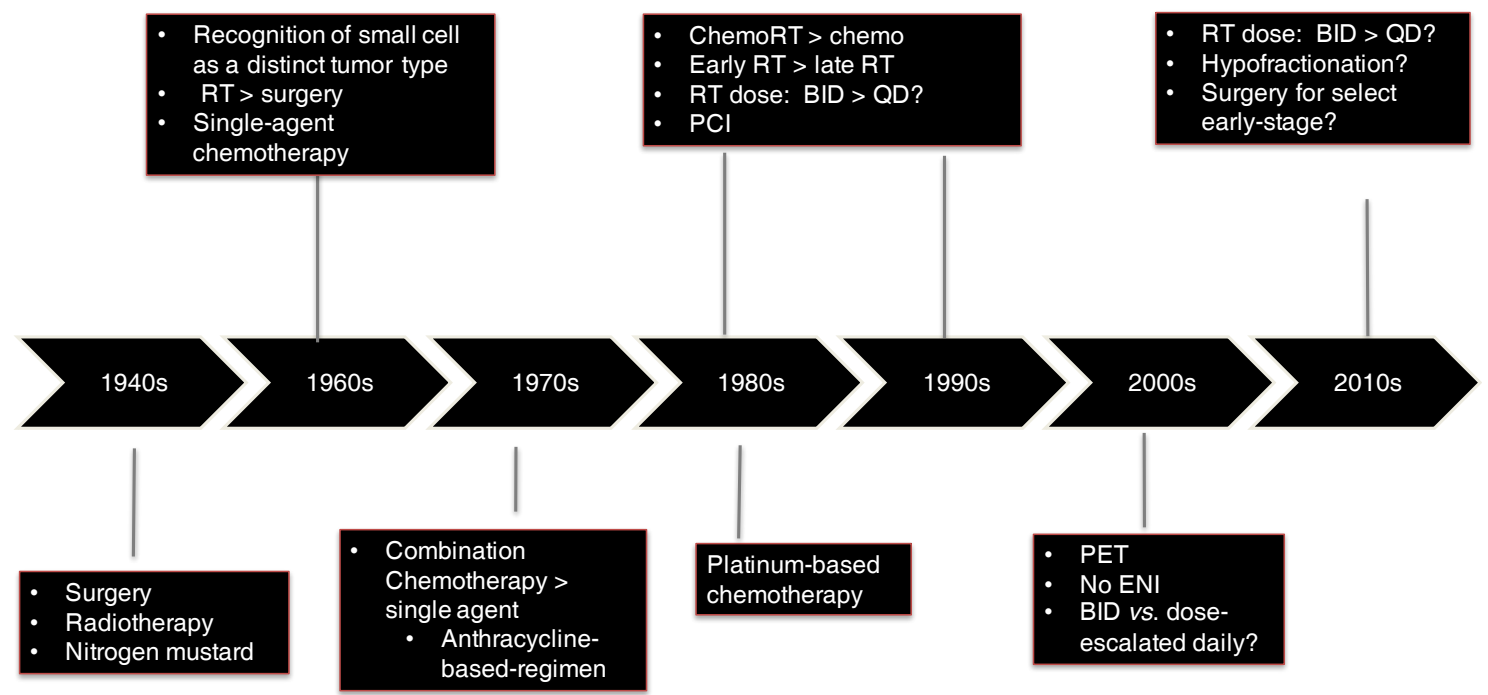

Figure 1 Management of small cell lung cancer. RT, radiotherapy; BID, twice a day; QD, 4 times a day; ENI, elective nodal irradiation.

initiated with cycle 2 of chemotherapy. Median survival time was improved to 21 months for patients treated with RT beginning at cycle 2 from 16 months for patients treated with RT beginning at cycle 6. A follow-up metaanalyses of all of studies done comparing early $v$ s. delayed RT, confirmed the benefit for early $v s$. late RT, with the magnitude of the benefit largest in patients treated with platinum-based chemotherapy (17).

The most controversial question related to thoracic radiation has related to defining the optimal $\mathrm{RT}$ fractionation for LS-SCLC. This was first addressed through the Intergroup 0096 study (18), where patients were randomized to receive either 4 cycles of cisplatin and etoposide with RT to a dose of 45 Gy delivered in 1.5-Gy twice-daily fractions vs. 45 Gy delivered in 1.8-Gy once daily fractions, which was the standard RT regimen at the time the study was conducted. PCI was suggested but not mandated for patients who achieved a complete response (CR) following CRT. RT was delivered using two-dimensional planning techniques and the RT target volumes in the study were gross disease, with the ipsilateral hilum, and bilateral mediastinum with a $1.5-\mathrm{cm}$ margin. Patients with a pleural effusion and/or contralateral hilar or supraclavicular disease were not eligible for the study. The primary endpoint of the study was 2-year OS, with the study powered to detect a $15 \%$ improvement in 2-year OS from $25 \%$ to $40 \%$.

Long-term results of the study demonstrated an improvement in several outcome measures following the use of twice-daily RT (18). There was a non-significant increase in patients achieving $\mathrm{CR}$, a trend towards improved local control, and a significant improvement in combined local and distant failures, favoring the twice-daily arm. Although initial reports of the study showed a non-significant trend towards improved OS (19), long-term follow-up published after a median follow-up of 8 years showed a significant increase in median survival time in the BID arm from 19 to 23 months. The study was powered to detect a $15 \%$ improvement in OS from $25 \%$ to $40 \%$, but ultimately showed 2-year OS was significantly increased from $41 \%$ to $47 \%$. Five-year OS was also significantly increased from $16 \%$ in the once-daily arm to $26 \%$ in the twice-daily arm. Improvements in survival following the use of twice daily RT were at the price of increased high-grade esophagitis. Grade 3 esophagitis in the once-daily arm was $11 \%$ compared with $27 \%$ in the BID arm.

\section{LS-SCLC: the modern story}

In spite of this level 1 evidence showing survival improvements following twice-daily RT, patterns of care studies have shown that the majority of patients in the US with LS-SCLC are treated with once-daily RT. Based on data published using the National Cancer Database (20), it is estimated that only $11 \%$ of patients in the US have been treated with twice-daily RT following the publication of the Intergroup 0096 study.

Although the reasons for this are multifactorial, these 
findings are thought to be largely reflective of changes in the RT planning and delivery techniques that occurred over the time period when the Intergroup study was initiated-1989—and ultimately published-1999. Over this 10 -year time period, the standard once-daily radiation dose increased from 45-50 to 60-70 Gy due to the advent of three-dimensional (3D) RT planning techniques. Many physicians hypothesized that the survival benefit seen in the Intergroup 0096 study was simply related to the higher biologic dose delivered that was delivered using twice-daily RT, compared to once-daily group (21).

A second change in care that occurred following the publication of the Intergroup study, was the routine use of PCI for patients with limited stage disease. Although the brain had long been recognized as a sanctuary site for SCLC, the role of PCI had not been clearly established at the time that the Intergroup study was initiated. Although studies had consistently shown a reduction in brain metastases, they had inconsistently shown an OS benefit, while also demonstrating potential for serious neurotoxicity. However, a large meta-analysis published the same year as the Intergroup study demonstrated that the use of PCI resulted in a $50 \%$ decrease in the incidence of brain metastases at 3 years, as well as a $5 \%$ improvement in OS in the subset of patients who experienced a CR to initial therapy (22) .

Although PCI was offered to patients who achieved a CR on the Intergroup study, it was not mandatory. The number of patients who received PCI in the Intergroup study was not reported in the initial study publication, making some question if the survival difference was due to a difference in use of PCI between study arms. However, in a followup editorial, the study PI reported that there were more patients in the once-daily arm than in the twice-daily arm that actually received PCI (18).

Other clinical practice changes that have occurred since the publication of the Intergoup study include the use of PET/CT for staging as well as the omission of elective nodal RT. PET/CT staging is now considered a standard part of the workup for patients with newly diagnosed SCLC. The use of a PET/CT as compared to a CT with contrast and bone scan has been shown to result in $17 \%$ of patients being upstaged from limited to extensive stage, as well as about $5 \%$ of patients being down-staged from extensive to limited stage (23). Additionally, multiple case series that have been published that demonstrate equivalent outcomes for patients treated with involved nodal therapy as compared to elective RT. For patients staged with a PET/
CT, the isolated nodal failure rate for patients who received involved nodal RT is in the range of $3-5 \%(24,25)$.

Therefore, the question that has remained is how twice-daily RT compares to dose-escalated once-daily RT delivered using modern RT techniques. The CONVERT study was designed to address this specific question (26). This was a multicenter study conducted in Europe and Canada, in which patients with LS-SCLC were randomized to receive either 45 Gy in 1.5-Gy twice daily fractions with RT vs. RT to a dose of $66 \mathrm{~Gy}$ in 33 fractions once daily fractions. RT in the study was initiated at cycle 2 of cisplatin/etoposide. Patients with no evidence of progression following thoracic RT underwent PCI. There was no elective nodal RT in this study, and $3 \mathrm{D}$ conformal radiotherapy (3D-CRT) and intensity-modulated RT (IMRT) were both allowed. The primary endpoint of this study was OS and the study was designed to detect a survival benefit of $12 \%$ at 2 years (from $44 \%$ in the twice-daily arm to $56 \%$ in the once-daily arm) (27).

There was no significant difference between the two arms in terms of OS (the primary endpoint), PFS, local control, or metastatic progression. Two-year OS was 51\% in the once-daily arm compared with $56 \%$ in the BID arm, but not significantly different. Radiation-related toxicities were also lower than expected, likely as a result of the use of modern RT techniques. Notably, there was no significant difference in rates of high-grade esophagitis between both study arms. There was, however, a small but significant, increase in acute neutropenia in the twice-daily arm. One patient in the study - in the once daily arm-developed an esophageal stricture. Given that the CONVERT study was not designed as an equivalence study, the study authors concluded that twice-daily RT should be considered the standard of care, with once-daily RT considered for patients who decline twice-daily RT (27). Some important differences were noted between the two study arms: (I) a higher percentage of patients in the twice-daily compared to the once-daily arm were able to receive the full dose of RT (98\% vs. $83 \%$ ); and (II) a higher percentage of patients in the twice-daily versus the once-daily arm were able to complete their RT without significant treatment interruptions.

Another randomized study conducted in Europe to evaluate outcomes for patients treated with dose-escalated hypofractionated RT (28). This was a phase II study in which patients were randomized to receive either 45 Gy at 1.5 Gy delivered twice-daily or 42 Gy in 2.8 Gy once daily fractions. The primary endpoint was 1-year PFS and was 
powered to detect an improvement in PFS from $70 \%$ to $91 \%$. The results showed no statistical differences between the 2 arms in terms of PFS or OS: 1-year PFS was $45 \%$ vs. $49 \%$ in favor of the once daily arm; 2-year OS between the hypofractionated $v s$. twice daily arm was $42 \%$ vs. $53 \%$ and median OS time of $18 v$ s. 25 months, respectively. Grade 3 and 4 esophagitis rates were the same. A follow-up study is currently underway in Norway comparing 45 Gy at 1.5 Gy BID to 60 Gy at 1.5 Gy twice daily. The primary endpoint for this study is 2-year OS.

Therefore, the current standard of care for LS-SCLC is definitive chemoradiation, with RT initiated during cycle 1 or 2 of chemotherapy. Patients with no evidence of progression following thoracic treatment should receive PCI. Accelerated hyperfractionated RT (45 Gy in 1.5 Gy twice daily fractions) is the only $\mathrm{RT}$ regimen that has been shown to be superior to another RT regimen, and is the preferred fractionation scheme for patients with limited stage disease. However, it should be noted that there is a cooperative group study in the US (CALGB 30610), comparing the Turrisi regimen to 70 Gy of RT delivered in 2 Gy once daily fractions that is still ongoing.

\section{RT in extensive-stage SCLC (ES-SCLC)}

Recent studies have been conducted to further define the role of RT for PCI as well as consolidative extra-cranial RT in patients with ES-SCLC.

\section{PCI}

The Auperin meta-analysis, which demonstrated a $5 \%$ survival benefit at 3 years following PCI for patients with LS-SCLC (22), led to questions about the role of PCI in patients with extensive-stage disease who have an even higher risk of brain metastases. This was first addressed through a pivotal EORTC study in which patients with extensive-stage disease were randomized to receive PCI or observation following completion of systemic therapy (29). Patient with any response to systemic therapy were eligible for this clinical trial. The primary endpoint of the study was time to symptomatic brain metastases. Brain imaging with a CT or MRI was not required at staging or during follow-up, unless patients had symptoms suggestive of brain metastases. The most common RT regimen was 20 Gy in 5 fractions. The results of this study demonstrated: (I) the use of PCI was associated with a reduced risk of symptomatic brain metastasis (14.6\% vs. 40\%); (II) patients receiving PCI were more likely to remain healthy enough to receive second- or third-line chemotherapy ( $68 \%$ vs. $45 \%$ ); and (III) the use of PCI was also associated with a survival benefit $(27 \% v s$. $13.3 \%$ at 1 -year). In spite of these promising results, some have hypothesized that the advantages of PCI shown in the EORTC were partially due to the lack of brain imaging prior to treatment. If a significant portion of patients in the study had sub-clinical brain lesions prior to randomization, this would have resulted in a portion of patients in the PCI arm who received treatment asymptomatic brain metastases in the PCI arm compared to patients with untreated brain metastases in the observation arm.

This led to a large study conducted in Japan in which patients with extensive disease (with initial response to systemic therapy) were randomized to PCI or observation following systemic therapy (30). In contrast to the EORTC study, all patients underwent a re-staging MRI after chemotherapy. In addition, patients underwent an MRI every 3 months during the first year of follow-up and at 18 and 25 months following treatment. Patients who developed brain metastases in follow-up underwent RT at that time. This study again showed that PCI is very effective in reducing the risk of brain metastases (48\% vs. 69\%), but did not demonstrate a difference in OS. A pre-planned interim analysis of the Japanese study showed that the chance of PCI resulting in improved survival was $0.011 \%$ and the study was subsequently terminated.

There are several important differences between the EORTC study and Japanese study that are worth noting. In addition to different recommendations of MRI surveillance, patients in the EORTC study were only recommended treatment if they developed symptomatic brain metastases, as compared to the Japanese study where patients with radiographic evidence of brain metastases, including asymptomatic brain metastases, received salvage treatment. There were also significant differences in the OS of the patent cohorts between the two studies: in the EORTC study, the best arm had a $27 \%$ 1-year survival compared to nearly $50 \%$ in the Japanese study, raising the possibility that these studies included a very different group of patients. Moreover, patients who had PCI in the Japanese study were less likely to undergo

second and third-line chemotherapy, while the EORTC study demonstrated the opposite result. It should be stressed that the design of the Japanese trial differed from the EORTC study in that it was a non-inferiority trial that was underpowered for OS given that the study was terminated early (31). 
Taken together, these data demonstrate unequivocal evidence that PCI is associated with a significant reduction in the incidence of brain metastases in ES-SCLC. However, the benefit of PCI on OS may be debatable if patients receive routine surveillance imaging with an MRI brain. Moreover, the majority of patients who do not receive PCI will require salvage whole-brain RT (WBRT) for treatment of brain metastases.

\section{Thoracic radiation}

Following systemic therapy and the potential use of PCI, 85-90\% of ES-SCLC patients experience disease progression within the thorax (29). The value of consolidative thoracic therapy was first evaluated in a singleinstitution study conducted in Yugoslavia (32). This study evaluated a highly selected group of patients with extensivestage disease who achieved a CR at all distant sites of disease and CR or partial response (PR) within the thorax. Patients were then randomized to receive accelerated hyperfractionated RT to 54 Gy with daily carboplatin/ etoposide. Long-term results from this study showed an improvement in median survival time for patients receiving thoracic radiation (11 vs. 17 months).

The CREST study was later initiated in Europe to evaluate the role of consolidative thoracic RT for patients with no progression after initial chemotherapy in the setting of a multi-institutional study (33). Patients in this study were randomized to receive PCI with or without consolidative thoracic RT to 30 Gy in 10 fractions. Local intra-thoracic failure was significantly reduced from $80 \%$ to $44 \%$ with the addition of thoracic RT. A large proportion of patients in both study arms experienced progression at sites outside the brain and thorax. The difference in OS, the primary endpoint of the study, did not reach statistical significance $(\mathrm{P}=0.066)$. However, for longer-term survivors, there was a statistically significant OS benefit at 2 years favoring patients who received thoracic RT (13\% vs. $3 \%$ at 2 years). In a post-hoc analysis of the study comparing outcomes for patients who experienced a CR vs. PR in the thorax after systemic therapy, it was found that patients with PR derived the biggest benefit (34). Another secondary analysis of the study showed that patients with less than 3 sites of distant metastatic disease experienced the largest benefit from thoracic RT (35).

The NRG/RTOG Oncology Cooperative Group initiated RTOG 0937 in the US to evaluate the role of extracranial consolidative RT in ES-SCLC patients at the same time that the CREST study initiated in Europe (36). This study included patients with extensive-stage disease with 1-4 sites extracranial metastatic disease who achieved a PR/CR to systemic therapy. Patients with brain metastases were excluded. Patients were randomized to receive PCI with or without RT to the thorax as well as sites of distant metastatic disease. In contrast to the CREST study, patients were recommended to receive a dose of 45 Gy in 3 Gy fractions to the thorax (compared to $30 \mathrm{~Gy}$ in CREST) and 30-45 Gy to sites of distant disease. The primary endpoint of the study was OS and planned accrual was 154 patients. However, the study accrued very slowly and was terminated early after 97 evaluable patients were enrolled to the study due to a pre-planned interim analysis that showed the study crossed the futility boundary for OS. One-year OS was better than expected in both arms in both arms: $60.1 \%$ in the PCI arm and $50.8 \%$ in the PCI + RT arm (compared to $27 \%$ in the best arm of EORTC PCI study). Time to progression showed a significant benefit for the patients who received local RT. Pattern of failure analysis demonstrated that consolidative RT to all sites of extracranial disease following PCI reduced the risk of first failure in the thorax from $62.5 \%$ to $25.8 \%$ and reduced the risk of failure at one of the sites of presenting metastatic disease from $78.1 \%$ to $41.9 \%$.

Thus, while both the CREST and RTOG 0937 demonstrate a role for consolidative thoracic RT for ESSCLC patients with no progression after chemotherapy (especially for residual thoracic tumor), the RTOG shows no role for consolidative RT to sites of initial distant metastatic disease.

\section{Novel RT treatment approaches for management of SCLC}

\section{Reducing neurocognitive dysfunction following PCI}

While PCI has been shown to reduce the incidence of brain metastases, and in some situations improve OS, this comes at a price of potential neurocognitive toxicity that may significantly impact quality of life. In RTOG 0212, a randomized study evaluating high-dose $v s$. standard-dose PCI, high-dose PCI was associated with a $25 \%$ absolute increase in the rate of chronic cognitive toxicity (37-39). However, even in the subgroup of patients receiving standard-dose PCI (25 Gy/10 fx), 62\% developed cognitive toxicity as measured by the Hopkins verbal learning test (HVLT) (4). Moreover, in a meta-analysis of the 2 RTOG studies evaluating PCI, it was observed that PCI relative to 
observation leads to a decline not only in the HVLT but also in self-reported cognitive functioning as tested by the EORTC QLQ-C30 at 6 and 12 months after treatment (40). Taken together and combined with similar data from the setting of therapeutic WBRT for brain metastases, these data suggest that neurocognitive dysfunction is an adverse effect of cranial irradiation, that involves an exquisite differential sensitivity of memory-related domains over other neurocognitive domains and that is pertinent to patient-reported quality of life. Patterns of care studies have shown that only $60 \%$ of patients with SCLC treated at an academic center receive PCI, largely due to concerns about cognitive toxicity (41).

The primary method for reducing memory impairment from WBRT has been use of stereotactic radiosurgery (SRS) in place of WBRT. However, most clinical trials of SRS have excluded patients with SCLC. Small, singleinstitution retrospective studies have been published $(42,43)$, but no firm conclusion can be made from these studies given the small number of patients included in the analyses. Moreover, even with PCI, a $30-50 \%$ of patients will develop new or progressive brain metastases, highlighting the need for WBRT in this patient population.

Recent emphasis has been placed on delivering WBRT with hippocampal avoidance (HA) using IMRT to reduce memory impairment from PCI. A recently conducted phase II study by the RTOG demonstrated the feasibility of delivering WBRT with conformal avoidance of the hippocampus (HA-WBRT) for patients with brain metastases in a cooperative group setting (44). The primary endpoint was HVLT assessment of delayed recall (DR) at 4 months. Results of the study demonstrated favorable rates memory preservation following HA-WBRT. The mean relative decline in HVLT-DR from baseline to 4 months was $7.0 \%$ (95\% CI: $-4.7 \%$ to $18.7 \%$ ), which was significantly improved compared to the pre-specified historical control of $30 \%$ for the same period (45). Moreover, there was no significant decline in any of the measured quality of life domains.

A single-institution study conducted at Johns Hopkins University prospectively evaluated the role of HA for patients with SCLC receiving PCI (46). The primary endpoint of the study was the HVLT-DR test measured at 6 months. The study target accrual was 125 patients, but closed early to support a cooperative group study evaluating HA-PCI. The analysis of the 20 patients enrolled on the study showed a mean decline on the HVLT of 0.38 , which was improved over the mean decline of 1.0 as reported in RTOG 0212. However, two patients developed brain metastases within the HA volume. Both patients were also found to have one or more brain metastases in fully treated brain regions.

An ongoing cooperative group study, NRG CC003, is currently being conducted to better define the role of HA in SCLC patients undergoing PCI. This is a phase II/ III study in which patients with limited or extensive-stage disease are randomized to receive PCI with or without HA. The primary objective of the phase II study, which completed accrual in October 2017, is to establish the noninferiority of HA in terms of intracranial relapse. If noninferiority of HA is established, the phase III component of the study will compare neurocognitive outcomes of patients using the HVLT-DR at 6 months as the primary outcome measure. Although the use of HA would increase costs of treatment, a cost-effectiveness analysis shows this treatment approach to be cost-effective if the results of NRG CC003 are positive (47).

\section{Stereotactic body RT (SBRT)}

Patients with early stage SCLC represent a small but significant subset of patients with newly diagnosed SCLC. The National Lung Screening Trial, which showed a 20\% relative reduction in lung cancer mortality and an almost $7 \%$ reduction in all-cause cancer mortality, found that $7 \%$ of CT screen detected lung cancer cases were SCLC and observed an increase in the detection of early-stage SCLC (48). The impact of CT screening on survival of SCLC is unclear, but the optimal management of these patients is still controversial. Recently published surgical series show promising results, which have been endorsed by the National Comprehensive Cancer Network (49).

SBRT is the preferred treatment option for patients with early-stage inoperable NSCLC (50). Until recently, there was little evidence to support the use of SBRT in patients with early-stage SCLC. However, a recent multi-institutional retrospective analysis described outcomes of 74 early-stage SCLC patients from 24 institutions treated with SBRT (51). With a median follow-up time of 18 months, the local control rate was $97 \%$ at 1 year and $96 \%$ at 3 years, respectively. PFS at those time points was $58 \%$ and $53 \%$, respectively, and OS was $70 \%$ at 1 year and $34 \%$ at 3 years. Grade 2 pneumonitis risk was $5 \%$. The predominant failure pattern was distant, which was observed in $46 \%$ of patients. There was a $10 \%$ risk of isolated nodal failure. Receipt of chemotherapy following SBRT was significantly associated with an improved PFS 
and OS. Based on this retrospective series, SBRT followed by chemotherapy may be a safe treatment option for patients who are not candidates for definitive chemoradiation or surgery followed by chemotherapy.

\section{Proton beam therapy}

Emerging evidence from NSCLC has demonstrated a significant correlation between RT dose to the heart and OS, even after correcting for other well-established prognostic factors (52). This has led to interest in evaluating the role of proton beam therapy as a method to reduce RT dose to the heart while maintaining definitive RT dose required for lung cancer treatment. Although this relationship is best established in NSCLC, it is plausible that a similar phenomenon is seen in SCLC patients, where patients are even more likely to have bulky central disease that will result in higher RT doses to the heart using photon radiotherapy.

The results of a prospective study evaluating outcomes for LS-SCLC patients tread with proton therapy were recently published (53). This single-institution study from the University of Pennsylvania included 30 patients treated to a median dose of 63.9 Gy cobalt Gy equivalents. The median OS for the cohort was 28.2 months and there few high-grade treatment-related adverse events. Although the results of this prospective study are encouraging, they require validation in future clinical trials.

\section{Conclusions}

RT plays a central role in the management of SCLC. Patients with limited stage disease should receive definitive chemoradiation followed by PCI for any patients with no evidence of progression following initial thoracic therapy. Data continues to support the use of accelerate hyperfractionation compared to conventionally fractionated RT. Patients with extensive-stage disease also benefit from PCI and consolidative RT; however, given the worse prognosis of these patients, treatment recommendations should incorporate patient preferences for treatment intensification and quality of life. Newer RT approaches involving HA, SBRT, and proton beam therapy provide hope for reducing RT treatment-related morbidities, pending further evaluation in prospective clinical trials.

\section{Acknowledgements}

None.

\section{Footnote}

Conflicts of Interest: The authors have no conflicts of interest to declare.

\section{References}

1. Siegel RL, Miller KD, Jemal A. Cancer Statistics, 2018. CA Cancer J Clin 2018;68:7-30.

2. Watson WL, Berg JW. Oat cell lung cancer. Cancer 1962;15:759-68.

3. Chang A, Pfeifer K, Chen P, et al. Thoracic Presentations of Small Round Blue Cell Tumors. J Clin Imaging Sci 2016;6:25.

4. Jackman DM, Johnson BE. Small-cell lung cancer. Lancet 2005;366:1385-96.

5. Alberg AJ, Shopland DR, Cummings KM. The 2014 Surgeon General's report: commemorating the 50th Anniversary of the 1964 Report of the Advisory Committee to the US Surgeon General and updating the evidence on the health consequences of cigarette smoking. Am J Epidemiol 2014;179:403-12.

6. Thun MJ, Carter BD, Feskanich D, et al. 50-year trends in smoking-related mortality in the United States. N Engl J Med 2013;368:351-64.

7. Wang S, Tang J, Sun T, et al. Survival changes in patients with small cell lung cancer and disparities between different sexes, socioeconomic statuses and ages. Sci Rep 2017;7:1339.

8. Zelen M. Keynote address on biostatistics and data retrieval. Cancer Chemother Rep 3 1973;4:31-42.

9. Comparative trial of surgery and radiotherapy for the primary treatment of small-celled or oat-celled carcinoma of the bronchus. First report to the Medical Research Council by the working-party on the evaluation of different methods of therapy in carcinoma of the bronchus. Lancet 1966;2:979-86.

10. Miller AB, Fox W, Tall R. Five-year follow-up of the Medical Research Council comparative trial of surgery and radiotherapy for the primary treatment of smallcelled or oat-celled carcinoma of the bronchus. Lancet 1969;2:501-5.

11. Chan BA, Coward JI. Chemotherapy advances in smallcell lung cancer. J Thorac Dis 2013;5 Suppl 5:S565-78.

12. Perry MC, Eaton WL, Propert KJ, et al. Chemotherapy with or without radiation therapy in limited small-cell carcinoma of the lung. N Engl J Med 1987;316:912-8.

13. Thomas CR Jr, Giroux DJ, Janaki LM, et al. Ten-year 
follow-up of Southwest Oncology Group 8269: a phase II trial of concomitant cisplatin-etoposide and daily thoracic radiotherapy in limited small-cell lung cancer. Lung Cancer 2001;33:213-9.

14. Pignon JP, Arriagada R, Ihde DC, et al. A meta-analysis of thoracic radiotherapy for small-cell lung cancer. N Engl J Med 1992;327:1618-24.

15. Warde P, Payne D. Does thoracic irradiation improve survival and local control in limited-stage small-cell carcinoma of the lung? A meta-analysis. J Clin Oncol 1992;10:890-5.

16. Murray N, Coy P, Pater JL, et al. Importance of timing for thoracic irradiation in the combined modality treatment of limited-stage small-cell lung cancer. The National Cancer Institute of Canada Clinical Trials Group. J Clin Oncol 1993;11:336-44.

17. Pijls-Johannesma M, De Ruysscher D, Vansteenkiste J, et al. Timing of chest radiotherapy in patients with limited stage small cell lung cancer: a systematic review and metaanalysis of randomised controlled trials. Cancer Treat Rev 2007;33:461-73.

18. Turrisi AT 3rd, Kim K, Blum R, et al. Twice-daily compared with once-daily thoracic radiotherapy in limited small-cell lung cancer treated concurrently with cisplatin and etoposide. N Engl J Med 1999;340:265-71.

19. Wagner H, Kim K, Johnson DH, et al. Daily (D) vs. twice-daily (BID) thoracic irradiation (TI) with concurrent cisplatinietoposide (PE) chemotherapy as initial therapy for patients with limited small cell lung cancer (LSCLC): Preliminary results of a phase III prospective intergroup trial. Int J Radiat Oncol Biol Phys 1994;30:178.

20. Schreiber D, Wong AT, Schwartz D, et al. Utilization of Hyperfractionated Radiation in Small-Cell Lung Cancer and Its Impact on Survival. J Thorac Oncol 2015;10:1770-5.

21. Abadir R, Orton C. Radiotherapy for small-cell lung cancer. N Engl J Med 1999;340:2002-3; author reply 2003-4.

22. Auperin A, Arriagada R, Pignon JP, et al. Prophylactic cranial irradiation for patients with small-cell lung cancer in complete remission. Prophylactic Cranial Irradiation Overview Collaborative Group. N Engl J Med 1999;341:476-84.

23. Kalemkerian GP. Staging and imaging of small cell lung cancer. Cancer Imaging 2012;11:253-8.

24. Shirvani SM, Komaki R, Heymach JV, et al. Positron emission tomography/computed tomography-guided intensity-modulated radiotherapy for limited-stage small-cell lung cancer. Int J Radiat Oncol Biol Phys 2012;82:e91-7.

25. van Loon J, De Ruysscher D, Wanders R, et al. Selective nodal irradiation on basis of (18)FDG-PET scans in limited-disease small-cell lung cancer: a prospective study. Int J Radiat Oncol Biol Phys 2010;77:329-36.

26. Faivre-Finn C, Falk S, Ashcroft L, et al. Protocol for the CONVERT trial-Concurrent ONce-daily VErsus twicedaily RadioTherapy: an international 2-arm randomised controlled trial of concurrent chemoradiotherapy comparing twice-daily and once-daily radiotherapy schedules in patients with limited stage small cell lung cancer (LS-SCLC) and good performance status. BMJ Open 2016;6:e009849.

27. Faivre-Finn C, Snee M, Ashcroft L, et al. Concurrent once-daily versus twice-daily chemoradiotherapy in patients with limited-stage small-cell lung cancer (CONVERT): an open-label, phase 3, randomised, superiority trial. Lancet Oncol 2017;18:1116-25.

28. Gronberg BH, Halvorsen TO, Flotten O, et al. Randomized phase II trial comparing twice daily hyperfractionated with once daily hypofractionated thoracic radiotherapy in limited disease small cell lung cancer. Acta Oncol 2016;55:591-7.

29. Slotman B, Faivre-Finn C, Kramer G, et al. Prophylactic cranial irradiation in extensive small-cell lung cancer. $\mathrm{N}$ Engl J Med 2007;357:664-72.

30. Takahashi T, Yamanaka T, Seto T, et al. Prophylactic cranial irradiation versus observation in patients with extensive-disease small-cell lung cancer: a multicentre, randomised, open-label, phase 3 trial. Lancet Oncol 2017;18:663-71.

31. Schild SE, Sio TT, Daniels TB, et al. Prophylactic Cranial Irradiation for Extensive Small-Cell Lung Cancer. J Oncol Pract 2017;13:732-8.

32. Jeremic B, Shibamoto Y, Nikolic N, et al. Role of radiation therapy in the combined-modality treatment of patients with extensive disease small-cell lung cancer: A randomized study. J Clin Oncol 1999;17:2092-9.

33. Slotman BJ, van Tinteren H, Praag JO, et al. Use of thoracic radiotherapy for extensive stage small-cell lung cancer: a phase 3 randomised controlled trial. Lancet 2015;385:36-42.

34. Slotman BJ, van Tinteren H. Which patients with extensive stage small-cell lung cancer should and should not receive thoracic radiotherapy? Transl Lung Cancer Res 2015;4:292-4.

35. Slotman BJ, Faivre-Finn C, van Tinteren H, et al. Which 
patients with ES-SCLC are most likely to benefit from more aggressive radiotherapy: A secondary analysis of the Phase III CREST trial. Lung Cancer 2017;108:150-3.

36. Gore EM, Hu C, Sun AY, et al. Randomized Phase II Study Comparing Prophylactic Cranial Irradiation Alone to Prophylactic Cranial Irradiation and Consolidative Extracranial Irradiation for Extensive-Disease Small Cell Lung Cancer (ED SCLC): NRG Oncology RTOG 0937. J Thorac Oncol 2017;12:1561-70.

37. Le Pechoux C, Dunant A, Senan S, et al. Standard-dose versus higher-dose prophylactic cranial irradiation (PCI) in patients with limited-stage small-cell lung cancer in complete remission after chemotherapy and thoracic radiotherapy (PCI 99-01, EORTC 22003-08004, RTOG 0212, and IFCT 99-01): a randomised clinical trial. Lancet Oncol 2009; 10:467-74.

38. Le Pechoux C, Laplanche A, Faivre-Finn C, et al. Clinical neurological outcome and quality of life among patients with limited small-cell cancer treated with two different doses of prophylactic cranial irradiation in the intergroup phase III trial (PCI99-01, EORTC 22003-08004, RTOG 0212 and IFCT 99-01). Ann Oncol 2011;22:1154-63.

39. Wolfson AH, Bae K, Komaki R, et al. Primary analysis of a phase II randomized trial Radiation Therapy Oncology Group (RTOG) 0212: impact of different total doses and schedules of prophylactic cranial irradiation on chronic neurotoxicity and quality of life for patients with limiteddisease small-cell lung cancer. Int J Radiat Oncol Biol Phys 2011;81:77-84.

40. Gondi V, Paulus R, Bruner DW, et al. Decline in tested and self-reported cognitive functioning after prophylactic cranial irradiation for lung cancer: pooled secondary analysis of Radiation Therapy Oncology Group randomized trials 0212 and 0214. Int J Radiat Oncol Biol Phys 2013;86:656-64.

41. Lok BH, Ma J, Foster A, et al. Factors influencing the utilization of prophylactic cranial irradiation in patients with limited-stage small cell lung cancer. Adv Radiat Oncol 2017;2:548-54.

42. Wegner RE, Olson AC, Kondziolka D, et al. Stereotactic radiosurgery for patients with brain metastases from small cell lung cancer. Int J Radiat Oncol Biol Phys 2011;81:e21-7.

43. Yomo S, Hayashi M. Upfront stereotactic radiosurgery in patients with brain metastases from small cell lung cancer: retrospective analysis of 41 patients. Radiat Oncol 2014;9:152.
44. Gondi V, Pugh SL, Tome WA, et al. Preservation of memory with conformal avoidance of the hippocampal neural stem-cell compartment during whole-brain radiotherapy for brain metastases (RTOG 0933): a phase II multi-institutional trial. J Clin Oncol 2014;32:3810-6.

45. Mehta MP, Rodrigus P, Terhaard CH, et al. Survival and neurologic outcomes in a randomized trial of motexafin gadolinium and whole-brain radiation therapy in brain metastases. J Clin Oncol 2003;21:2529-36.

46. Redmond KJ, Hales RK, Anderson-Keightly H, et al. Prospective Study of Hippocampal-Sparing Prophylactic Cranial Irradiation in Limited-Stage Small Cell Lung Cancer. Int J Radiat Oncol Biol Phys 2017;98:603-11.

47. Qu XM, Mishra MV, Bauman GS, et al. Cost-effectiveness of prophylactic cranial irradiation with hippocampal avoidance in limited stage small cell lung cancer. Radiother Oncol 2017;122:411-5.

48. National Lung Screening Trial Research T, Aberle DR, Adams AM, et al. Reduced lung-cancer mortality with low-dose computed tomographic screening. N Engl J Med 2011;365:395-409.

49. Barnes H, See K, Barnett S, et al. Surgery for limitedstage small-cell lung cancer. Cochrane Database Syst Rev 2017;4:CD011917.

50. Timmerman R, Paulus R, Galvin J, et al. Stereotactic body radiation therapy for inoperable early stage lung cancer. JAMA 2010;303:1070-6.

51. Verma V, Shostrom VK, Kumar SS, et al. Multiinstitutional experience of stereotactic body radiotherapy for large ( $\geq 5$ centimeters) non-small cell lung tumors. Cancer 2017;123:688-96.

52. Bradley JD, Paulus R, Komaki R, et al. Standard-dose versus high-dose conformal radiotherapy with concurrent and consolidation carboplatin plus paclitaxel with or without cetuximab for patients with stage IIIA or IIIB non-small-cell lung cancer (RTOG 0617): a randomised, two-by-two factorial phase 3 study. Lancet Oncol 2015;16:187-99.

53. Rwigema JM, Verma V, Lin L, et al. Prospective study of proton-beam radiation therapy for limited-stage small cell lung cancer. Cancer 2017;123:4244-51.

Cite this article as: Mishra MV, Louie AV, Gondi V, Slotman B. The evolving role of radiotherapy in the management of small cell lung cancer. J Thorac Dis 2018;10(Suppl 21):S2545-S2554. doi: $10.21037 /$ jtd.2018.06.98 